\title{
Presencia de hábitos orales en pacientes con maloclusiones de 4 a 14 años, Cali, Colombia
}

\author{
Presence of oral habits in patients with malocclusions \\ of 4 to 14 years, Cali, Colombia
}
Presença de hábitos orais em pacientes com malocclusões de 4 a 14 anos, Cali, Colômbia

\section{Nataly Johanna Mora-Zuluaga ${ }^{1}$ Katherine Torres-Trujillo ${ }^{2}$ Natalia Aragón ${ }^{3}$ Libia Soto-Llanos ${ }^{4}$}

Recibido: 21 de septiembre de 2020 Aprobado: 26 de noviembre de 2020 Publicado: 30 de diciembre de 2020

Cómo citar este artículo: Mora-Zuluaga NJ, Torres-Trujillo K, Aragón N, Soto-Llanos L. Presencia de hábitos orales en pacientes con maloclusiones de 4 a 14 años, Cali, Colombia. Revista Nacional de

Odontología. (2020); 16(2), 1-12. doi: https://doi.org/10.16925/2357-4607.2020.02.04

Artículo de investigación. https://doi.org/10.16925/2357-4607.2020.02.04

1 Odontóloga, Esp en Odontología Pediátrica y Ortopedia Maxilar, docente auxiliar tiempo parcial, Escuela Odontología, Universidad del Valle. Colombia.

ORCID: https://orcid.org/0000-0002-2286-215X

2 Odontóloga, Esp en Odontología Pediátrica y Ortopedia Maxilar Universidad del Valle, Colombia.

ORCID: https://orcid.org/0000-0002-4311-285X

3 Odontóloga, $\mathrm{PhD}(\mathrm{c})$, docente asistente tiempo completo, Escuela de Odontología Universidad del Valle, Colombia.

ORCID: https://orcid.org/0000-0003-4261-306X

4 Odontóloga, Esp Odontopediatría y Ortodoncia Clínica, MsC Administración. Profesor titular Escuela de Odontología Universidad del Valle. Colombia . Calle 4b \#36-00. CaliColombia.

ORCID: https://orcid.org/0000-0002-3252-7558

Correo electrónico: libia.soto@correounivalle.edu.co 


\title{
Resumen
}

Introducción: Durante la etapa de crecimiento y desarrollo, los hábitos orales de los niños pueden causar la presencia de maloclusiones según la frecuencia, duración e intensidad y tipo de habito. En este contexto, el objetivo de este estudio fue describir la presencia de hábitos orales no fisiológicos y evaluar su relación con los diferentes tipos de maloclusión.

Métodos: Estudio descriptivo de corte transversal, se incluyeron 77 niños entre los 4 y 14 años, de 2018 a 2019. Se realizó un análisis univariado reportando frecuencia y un análisis bivariado para evaluar independencia de las variables aplicando la prueba de $\mathrm{Chi}^{2}$ Pearson.

Resultados: La distribución según el tipo de dentición fue temporal 20,8\%, mixta $71,4 \%$ y permanente 7,8\%. La presencia de hábitos predominó en mujeres. La interposición lingual fue el hábito más frecuente en pacientes con los tres tipos de maloclusión, seguido del rechinamiento nocturno para pacientes con maloclusión clase I. Aquellos con mordida profunda presentaron rechinamiento nocturno en un $16 \%$ y los de mordida abierta interposición lingual en un $54,4 \%$.

Conclusiones: Se encontró mayor frecuencia de hábitos orales no fisiológicos en pacientes en dentición mixta. No hubo relación estadística entre los hábitos orales y la presencia de maloclusión.

Palabras clave: hábitos orales; maloclusión; niños; adolescentes.

\begin{abstract}
Introduction: The presence of oral habits influences the development of malocclusions according on its frequency, duration, and intensity; especially during the growth and development stage. In this context, our objective with this study was to describe the presence of non-physiological oral habits and to evaluate its relationship with the different types of malocclusion.

Methods: A descriptive cross-sectional study was conducted in 77 children between 4 and 14 years old, from 2018 to 2019. A univariate analysis was performed through frequency tables and a bivariate analysis to assess the independence of the variables by applying the Pearson's $\mathrm{Chi}^{2}$ test.

Results: The distribution according to the type of dentition was temporary $20.8 \%$, mixed $71.4 \%$ and permanent $7.8 \%$. The presence of habits predominated in women. Tongue thrusting was the habit the most frequent habit for patients with the three types of malocclusion, followed by night grinding for patients with class I malocclusion. Those with a deep bite presented night grinding in $16 \%$ and those with an anterior open bite tongue thrusting in $54.4 \%$.
\end{abstract}

Conclusions: A higher frequency of non-physiological oral habits was found in patients with mixed dentition. There was no statistical relationship between oral habits and the presence of malocclusion.

Keywords: oral habits; malocclusion; children; adolescents.

\section{Resumo}

Introdução: a presença de hábitos orais influencia o desenvolvimento das más oclusões de acordo com sua frequência, duração e intensidade; especialmente durante a fase de crescimento e desenvolvimento. Nesse contexto, nosso objetivo com este estudo foi descrever a presença de hábitos orais não fisiológicos e avaliar sua relação com os diferentes tipos de maloclusão.

Métodos: Foi realizado um estudo transversal descritivo em 77 crianças entre 4 e 14 anos, de 2018 a 2019. Foi realizada análise univariada por meio de tabelas de frequência e análise bivariada para avaliar a independência das variáveis por meio da aplicação do teste Chi2 de Pearson . 
Resultados: A distribuição segundo o tipo de dentição foi temporária 20,8\%, mista $71,4 \%$ e permanente $7,8 \%$. A presença de hábitos predominou nas mulheres. 0 empurrão da língua foi o hábito mais frequente nos pacientes com os três tipos de maloclusão, seguido da ranger de língua noturna nos pacientes com má oclusão de classe I. Aqueles com mordida profunda apresentaram ranger noturno em $16 \%$ e aqueles com mordida aberta anterior empurrando a língua em $54,4 \%$.

Conclusões: Foi encontrada maior frequência de hábitos orais não fisiológicos em pacientes com dentição mista. Não houve relação estatística entre os hábitos orais e a presença de maloclusão.

Palavras-chave: hábitos orais; má oclusão; crianças; adolescentes.

\section{Introducción}

Según la Organización Mundial de Salud las maloclusiones son alteraciones bucales asociadas con diferentes factores de riesgo tanto genéticos como ambientales. Dentro de estos se encuentran la presencia de hábitos orales no fisiológicos, que pueden influir en el desarrollo de una maloclusión dependiendo de la frecuencia, duración e intensidad durante la etapa de crecimiento y desarrollo. Como consecuencia a esto se presentan cambios en los tejidos óseos y en la oclusión.(1,2) La maloclusión, por su parte, es definida como la mala relación entre los arcos dentarios en cualquiera de los planos de espacio o en la posición inadecuada de los dientes más allá de los límites normales. (3) Esta posición inadecuada se debe a una alteración morfológica y funcional de los componentes dentarios, óseos y musculares del sistema estomatognático. Pueden ser de tipo dental cuando se presenta una desviación de los dientes de su oclusión ideal, o esquelética si hay alteración en la posición, forma y tamaño de los maxilares.(4,5) Existen diferentes tipos de maloclusiones y se encuentran relacionadas con los tres planos del espacio. En el plano sagital se encuentra la maloclusión clase I, clase II en sus divisiones 1 y 2, clase III y la mordida cruzada anterior. En el plano vertical se presenta la mordida abierta que puede ser anterior o posterior, y la mordida profunda que es contraria a esta. Finalmente, en el plano transversal se presenta la mordida cruzada posterior unilateral o bilateral, y la mordida en tijera. $(4,6,7)$.

El hábito está definido como una práctica adquirida por la repetición frecuente del mismo acto, que ocurre conscientemente al principio, pero luego de manera inconsciente, y que genera satisfacción (8). Sin embargo, existen hábitos no fisiológicos, los cuales se pueden deber a múltiples factores tanto psicológicos como predisposiciones morfológicas.(9) Los hábitos orales no fisiológicos tienen un origen multifactorial asociado a factores primarios como reacciones automáticas que alteran el comportamiento normal en situaciones como frustración o estrés, y a factores secundarios asociados con el entorno intrafamiliar o escolar.(10) Los hábitos más reportados en 
la población infantil son: la interposición lingual dada por ubicar la lengua en medio de los dientes al momento de deglutir y que genera una deglución atípica, la succión digital de uno o varios dedos de la mano, activando la musculatura perioral, la succión labial o queilofagia descrita como un mordisqueo o succión del labio, generalmente el inferior, (11) la onicofagia definida como el hábito de cortar, incidir o roer las uñas con los dientes, (12)(14) el bruxismo o rechinamiento nocturno descrito como una serie de movimientos mandibulares no funcionales de forma voluntaria o involuntaria que involucra los dientes en contacto continuo, (13) y la respiración oral cuando a su vez la lengua se ubica en una posición descendente que permite la entrada del aire.(9) Cuando este tipo de hábitos se prolongan sin intervención, se pueden presentar una serie de alteraciones en las estructuras del sistema estomatognático como paladar profundo u ojival, prognatismo maxilar, retrognatismo mandibular, proinclinación de incisivos superiores, retroinclinación de los incisivos inferiores, un overjet aumentado, mordida abierta anterior o posterior, mordida cruzada posterior, interferencia de la secuencia normal de la erupción, hipotonía labial, persistencia de deglución infantil, problemas fonéticos, o puede generar una tendencia a maloclusión clase II/1, entre otros. (14)

Se ha encontrado una asociación de los hábitos con las maloclusiones y son de importancia considerable para el pronóstico del tratamiento. De acuerdo con la literatura, éstos deben eliminarse para asegurar un ambiente funcional adecuado para el crecimiento fisiológico, debido a que pueden interferir no solo con la posición de los dientes, sino especialmente con el patrón de crecimiento esquelético normal.(15) Por lo anterior, el objetivo del presente estudio fue evaluar la presencia de diferentes hábitos orales no fisiológicos y su relación con los tipos de maloclusión en los tres planos del espacio, en una población infantil de 4 a 14 años.

\section{Métodos}

\section{Selección y descripción de los participantes}

Se realizó un estudio descriptivo de corte transversal en niños en edades entre los 4 a 14 años que asistieron a las clínicas de (Anónimo) entre mayo del 2018 y abril del 2019. El tamaño de muestra se calculó a través de la diferencia de proporciones del $25 \%$ en el grupo de expuestos con corrección de Fleiss, un error aceptado del 5\% y un poder del $80 \%$. Se seleccionaron pacientes que presentaran algún tipo de maloclusión en cualquiera de los tres planos del espacio y la presencia o no de algún hábito oral. Se excluyeron pacientes que presentaran algún síndrome o enfermedad 
sistémica y/o que se encontraran en tratamiento de ortopedia maxilar o ya lo hubiesen recibido. Esta investigación fue aprobada por el Comité Institucional de Revisión de Ética Humana (Anónimo). Se solicito la aprobación de los padres para participar en el estudio mediante la firma del consentimiento informado.

\section{Información técnica}

La muestra final fue de 77 pacientes a los cuales se les aplico un instrumento de evaluación clínica realizado por las investigadoras que incluyó variables de identificación, de oclusión y de hábitos orales no fisiológicos. Entre las variables de identificación se tuvo edad y sexo. Las variables de oclusión incluyeron tipo de dentición, es decir, temporal, mixta o permanente, tipos de maloclusión como sagital - clase I, II/1-2, III y mordida cruzada anterior-, vertical -mordida abierta anterior, mordida profunda y mordida borde a borde-, y transversal - mordida cruzada posterior unilateral o bilateral y mordida en tijera-. En cuanto a las variables de hábitos orales no fisiológicos, se tuvo en cuenta la interposición lingual, succión digital, onicofagia, succión labial y rechinamiento nocturno. Para la recolección de datos se utilizó como instrumento un formulario de Google Form ${ }^{\circledR}$ (herramienta Google ${ }^{\circledR}$ ) que generó una base de datos en una hoja de cálculo de Google® en tiempo real.

\section{Análisis estadístico}

Se realizó un análisis univariado de los datos a través de tablas de frecuencia y gráficos usando Microsoft Excelß y un análisis bivariado para evaluar la significancia estadística entre los tipos de maloclusión evaluadas y la presencia de hábitos, por medio del software STATA 13® aplicando la prueba de Chi $^{2}$ de Pearson con un error aceptado del 5\%. En cuanto al índice Kappa intra e interobservador, las evaluadoras obtuvieron un nivel "muy bueno" con una puntuación de 0,81.

\section{Resultados}

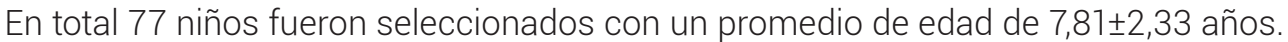
La distribución según el tipo de dentición fue: temporal 20,8\%, mixta 71,4\% y permanente $7,8 \%$. El $71 \%$ de la población presentó uno o más hábitos, de los cuales $65 \%$ fueron mujeres. La interposición lingual se presentó en un 44,2\%, seguido de la onicofagia en un 9,1\%, la succión digital en un 7,8\% y en menor porcentaje la succión labial y el rechinamiento nocturno, como se puede observar de manera detallada según el sexo en la Tabla 1. 
Tabla 1. Frecuencia de los tipos de hábitos en hombres y mujeres

\begin{tabular}{lccc}
\hline \multicolumn{1}{c}{ Hábito } & $\begin{array}{c}\text { Hombres } \mathbf{n}(\mathbf{\% )} \\
\mathbf{n = 2 8}\end{array}$ & $\begin{array}{c}\text { Mujeres } \mathbf{n}(\mathbf{\%}) \\
\mathbf{n = 4 9}\end{array}$ & $\begin{array}{c}\text { Total } \mathbf{n}(\mathbf{\%}) \\
\mathbf{n = 7 7}\end{array}$ \\
\hline Interposición lingual & $13(46,4)$ & $21(42,9)$ & $34(44,2)$ \\
\hline Onicofagia & $1(3,6)$ & $6(12,2)$ & $7(9,1)$ \\
\hline Succión labial & $2(7,1)$ & $2(4,1)$ & $4(5,2)$ \\
\hline Succión Digital & $1(3,6)$ & $5(10,2)$ & $6(7,8)$ \\
\hline Rechinamiento nocturno & $2(7,1)$ & $2(4,1)$ & $4(5,2)$ \\
\hline Ninguno & $9(32,1)$ & $13(26,5)$ & $22(28,6)$ \\
\hline
\end{tabular}

En relación con las maloclusiones y algún tipo de hábito, la interposición lingual fue predominante en todos los tipos de maloclusión en los tres planos del espacio, seguido de onicofagia en pacientes clase II división 1, III y en pacientes con mordida cruzada anterior; el rechinamiento nocturno en pacientes clase I y pacientes con mordida profunda; y la succión digital en pacientes con mordida cruzada posterior. El único paciente con mordida en tijera no presento ningún tipo de hábito. Esta distribución se presenta en la Tabla 2. El análisis bivariado de la relación entre la presencia de hábito con sexo y maloclusiones no arrojó ninguna relación de significancia estadística (Tabla 3).

Tabla 2. Frecuencia de tipo de maloclusión en relación con el tipo de habito presente en los niños.

\begin{tabular}{|c|c|c|c|c|c|c|c|c|}
\hline \multicolumn{2}{|c|}{ Maloclusión } & $n$ & $\begin{array}{c}\text { Interposición } \\
\text { lingual } \\
\mathbf{n}(\%)\end{array}$ & $\begin{array}{l}\text { Onicofagia } \\
\text { n (\%) }\end{array}$ & $\begin{array}{c}\text { Succión } \\
\text { labial } \\
\text { n (\%) }\end{array}$ & $\begin{array}{c}\text { Succión } \\
\text { digital } \\
\text { n (\%) }\end{array}$ & $\begin{array}{c}\text { Rechinamiento } \\
\text { nocturno } \\
\text { n (\%) }\end{array}$ & $\begin{array}{c}\text { Ninguno } \\
\mathbf{n}(\%)\end{array}$ \\
\hline \multirow{4}{*}{ Sagital } & Clase I & 30 & $11(36,7)$ & $1(3,3)$ & $2(6,7)$ & $3(10,0)$ & $4(13,3)$ & $9(30,0)$ \\
\hline & Clase II/1 & 33 & $17(51,5)$ & $4(12,1)$ & $2(6,1)$ & $3(9,1)$ & $0(0,0)$ & $7(21,2)$ \\
\hline & Clase II/2 & 3 & $1(33,3)$ & $0(0,0)$ & $O(0,0)$ & $0(0,0)$ & $0(0,0)$ & $2(66,7)$ \\
\hline & Clase III & 11 & $5(45,5)$ & $2(18,2)$ & $0(0,0)$ & $0(0,0)$ & $0(0,0)$ & $4(36,4)$ \\
\hline \multirow{3}{*}{ Vertical } & $\begin{array}{l}\text { Borde a } \\
\text { borde }\end{array}$ & 6 & $4(66,7)$ & $1(16,7)$ & $0(0,0)$ & $1(16,7)$ & $0(0,0)$ & $0(0,0)$ \\
\hline & $M P$ & 25 & $4(16,0)$ & $3(12,0)$ & $3(12,0)$ & $1(4,0)$ & $4(16.0)$ & $10(40,0)$ \\
\hline & MA & 11 & $6(54,4)$ & $0(0,0)$ & $0(0,0)$ & $3(27,3)$ & $0(0,0)$ & $2(18,2)$ \\
\hline \multirow{2}{*}{ Transversal } & MCP & 9 & $4(44,4)$ & $0(0,0)$ & $0(0,0)$ & $2(22,2)$ & $0(0,0)$ & $3(33,3)$ \\
\hline & MT & 1 & $0(0.0)$ & $0(0,0)$ & $0(0,0)$ & $0(0,0)$ & $0(0,0)$ & $1(100)$ \\
\hline \multicolumn{2}{|c|}{$\begin{array}{l}\text { Mordida Cruzada } \\
\text { Anterior }\end{array}$} & 6 & $3(50,0)$ & $2(33,3)$ & $0(0,0)$ & $0(0,0)$ & $0(0,0)$ & $1(16,7)$ \\
\hline
\end{tabular}

MP. Mordida profunda, MA: Mordida abierta, MCP. Mordida cruzada posterior, MT: Mordida en tijera. 
Tabla 3. Relación de presencia hábito con sexo y maloclusiones.

\begin{tabular}{lcc}
\hline \multirow{2}{*}{ Variables } & \multicolumn{2}{c}{ Presencia de hábito } \\
\cline { 2 - 3 } & OR & P \\
\hline Sexo (femenino) & 0,2750 & 0,600 \\
\hline Maloclusión sagital & 3,3664 & 0,330 \\
\hline Maloclusión vertical & 0,6499 & 0,885 \\
\hline Maloclusión transversal & 2,6955 & 0,260 \\
\hline Mordida cruzada anterior & 2,6028 & 0,107 \\
\hline
\end{tabular}

\section{Discusión}

En el presente estudio fue alta la detección de hábitos orales no fisiológicos en pacientes con maloclusión. Las maloclusiones están asociadas a diferentes factores entre ellos, la presencia de hábitos orales no fisiológicos. (1,5,9,16-18) Sin embargo, otros autores describen que, aunque los hábitos orales son un factor importante para la presencia de maloclusiones, existen otros factores de riesgo como los hereditarios y los traumáticos que también se deben considerar al momento del diagnóstico. $(3,19)$ Es importante tener en cuenta que los hábitos no fisiológicos pueden ser consecuencias de diferentes factores tanto socioeconómicos como emocionales y psicológicos. Entre estos factores se encuentra la depresión y ansiedad que pueden influir en desarrollo de algún habito en niños y adolescentes y puede estar asociado a alguna maloclusión. Recientemente se realizó un estudio donde los autores encontraron una asociación entre la presencia de hábitos bucales y la maloclusión en diferentes etapas del desarrollo oclusal tanto en niños como adolescentes, pudiendo estar influenciado por la ansiedad, aunque se necesitan más estudios para validad esta información (20). Este sería un factor importante para tener en cuenta al momento de la evaluación clínica.

Muchos de estos hábitos pueden causar fatiga y rigidez muscular, síntomas que son considerados desencadenantes de trastornos de la articulación temporomandibular en la población infantil, al momento de hacer el diagnóstico. (21) La presencia de hábitos orales no fisiológicos en pacientes con algún tipo de maloclusión, oscila entre el 47\% y el 96\% según reportes en diferentes investigaciones (1,3,9,12,22), rango en el que se encuentra el valor estimado en el presente estudio.

Uno de los hallazgos principales de este estudio fue la alta frecuencia del hábito de interposición lingual coincidiendo con investigaciones realizadas en poblaciones similares. $(1,11,23)$ Este hábito se presenta normalmente en el primer periodo de 
recambio dental, y si este hábito persiste en el tiempo, conlleva a la presencia de una deglución atípica, afectando no solo el proceso de deglución sino también la fonación, pudiendo mantenerse esta alteración hasta la edad adulta.(7) Adicionalmente, se encontró un reporte en la literatura de una relación significativa entre la presencia de la interposición lingual y el desarrollo de una maloclusión clase III.(17) Así mismo, otro estudio reciente, relaciona la presencia de este hábito con apiñamiento dental severo. (24) Con respecto a la onicofagia, es un hábito que se presenta con alta frecuencia, ocupando la segunda posición no solo en el presente estudio sino también en los realizados en otras investigaciones, en niños entre los 4 y los 12 años. $(3,17,25)$ Este hábito está asociado a un cambio social y psicológico, además, es de suma importancia evaluarlo al momento del examen clínico pues es uno de los más difíciles de erradicar.(10,12) Aunque los hábitos como la succión digital y labial fueron encontrados en un menor porcentaje en esta investigación, es importante resaltar que, de acuerdo con estudios previos, la succión digital presenta una relación directa con la presencia de mordida cruzada posterior y la mordida abierta anterior. $(17,25)$ Sin embargo, en el presente estudio no se obtuvo una significancia estadística, posiblemente relacionado con la diferencia del tamaño de muestra con los estudios anteriormente mencionados. Con respecto a la succión labial, esta tiene una frecuencia que oscila entre 5 y 16 \% según lo reportado en la literatura; $(15,26,27)$ a pesar que la frecuencia en nuestros estudio fue mucho menor la presencia de este hábito requiere de una mayor objetividad para su diagnóstico, lo que le puede dar mucha variabilidad al comparar con diferentes estudios. Al realizar el análisis bivariado no se encontró significancia estadística en cuanto a la relación entre los tipos de maloclusiones en los tres planos del espacio y la presencia de hábitos, probablemente debido al tamaño de la muestra. Sin embargo, el presente estudio sí coincide con otros en que la maloclusión sagital clase I fue la más frecuente.(3,15,17,25)

Según los hallazgos de una investigación publicada recientemente, muy pocos estudios realizan un análisis de los hábitos orales no fisiológicos y maloclusiones, sino que se enfocan en la evaluación de un hábito especifico principalmente relacionados con succión no nutritiva, por lo cual es necesario seguir realizando estudios en este campo de la investigación. (18) Adicionalmente, se sugiere el desarrollo de programas enfocados en la educación de la salud oral y la intervención temprana de los hábitos. (24) 


\section{Conclusiones}

Se puede concluir que la mayoría de los niños estudiados con maloclusiones, presentaron en el examen clínico al menos un hábito oral no fisiológico. La interposición lingual fue el hábito más frecuente para los pacientes con cualquier tipo de maloclusión. La mayoría de los hábitos orales se detectaron en pacientes en dentición mixta y de género femenino. No se encontró significancia estadística al evaluar la independencia de las variables relacionadas con maloclusión probablemente debido a las limitaciones en el tamaño de muestra, por lo que se recomienda realizar más estudios con un mayor tamaño de muestra.

\section{Conflicto de intereses}

No existe conflicto de intereses entre los autores. Los autores declaramos que somos independientes con respecto a las instituciones financiadoras y de apoyo, y que durante la ejecución del trabajo o la redacción del manuscrito no hemos incidido intereses o valores distintos a los que usualmente tiene la investigación.

\section{Fuente de financiación}

Ninguna

\section{Referencias}

1. Mendoza Oropeza L, Meléndez Ocampo AF, Ortiz Sánchez R, Fernández López A. Prevalencia de las maloclusiones asociada con hábitos bucales nocivos en una muestra de mexicanos. Rev Mex Ortod. 2014;2(4):220-7. doi: https://doi.org/10.1016/S2395-9215(16)30038-1

2. OMS. Encuestas de salud bucodental. 4 ed. Ginebra: Métodos Básicos. 1997.

3. Hamoud Nafea Alanazi A, Fahad al Musayyab F, Anad Alshraray Y, Abdullah Aljuwaid H. Prevalence of Malocclusion and Its Association with Deleterious Oral Habits in Saudi School Children. Egypt J Hosp Med [Internet]. 2018;71(6):3290-4. doi: https://doi. org/10.12816/0047246 
4. Zhou Z, Liu F, Shen S, Shang L, Shang L, Wang X. Prevalence of and factors affecting malocclusion in primary dentition among children in Xi'an, China. BMC Oral Health. 2016;16(91). doi: https://doi.org/10.1186/s12903-016-0285-x

5. Rapeepattana S, Thearmontree A, Suntornlohanakul S. Etiology of malocclusion and dominant orthodontic problems in mixed dentition: A cross-sectional study in a group of Thai children aged 8-9 years. J Int Soc Prev Community Dent. 2019;9(4):383. doi: https://doi. org/10.4103/jispcd.JISPCD_120_19

6. Thilander B, Bjerklin K, Lars B. Essential Orthodontics. Wiley; 2018.

7. Nascimento MHA, de Araújo TM, Machado AW. Severe Anterior Open Bite during Mixed Dentition Treated with Palatal Spurs. J Clin Pediatr Dent. 2016;40(3):247-50. doi: https://doi. org/10.17796/1053-4628-40.3.247

8. Diefenbacher S, Pfattheicher S, Keller J. On the Role of Habit in Self-Reported and Observed Hand Hygiene Behavior. Appl Psychol Heal Well-Being. 2020;12(1):125-43. doi: https://doi. org/10.1111/aphw.12176

9. Chamorro AF, García C, Mejía E, Viveros E, Soto L, Triana FE, et al. Hábitos orales frecuentes en pacientes del área de odontopediatría de la universidad del valle. Rev Gastrohnup. 2017;140:17-25.

10. Leme M, Barbosa T, Castelo P, Gavião M. Associations between Psychological Factors and the Presence of Deleterious Oral Habits in Children and Adolescents. J Clin Pediatr Dent. 2014;38(4):313-7. doi: https://doi.org/10.17796/jcpd.38.4.c48238322205466w

11. Agrawal S, Koirala B, Dali M, Shrestha S, Bhagat T, Niraula SR. Prevalence of Oral Habits of Schoolchildren in the Mixed Dentition in Dharan, Nepal. J Dent Child. 2019;86(2):88-92.

12. Winebrake JP, Grover K, Halteh P, Lipner SR. Pediatric onychophagia: A survey-based study of prevalence, etiologies, and co-morbidities. Am J Clin Dermatol. 2018;19(6):887-91. doi: https://doi.org/10.1007/s40257-018-0386-1

13. Pinos Robalino PJ, Gonzabay Bravo EM, Cedeño Delgado MJ. El bruxismo conocimientos actuales. Una revisión de la literatura. Reciamuc. 2020;4(1):49-58. doi: 10.26820/reciamuc/4. (1).enero.2020.49-58

14. Pipa Vallejo A, Cuerpo García de los Reyes P, López-Arranz Monje E, González García M, Pipa Muñiz I, Acevedo Prado A. Prevalencia de maloclusión en relación con hábitos de succión no nutritivos en niños de 3 a 9 años en Ferrol. Av Odontoestomatol. 2011;27(3):137-45. 
15. López Gallegos E, Armas A, López Ríos E, Nuñez A, Tatés Almeida K. Prevalencia de hábitos deletéreos y maloclusiones en dentición mixta en niños de la ciudad de Quito, Ecuador. KIRU. 2018;15(3).

16. Prada DC, Gamboa ERO, Murillo EAT. Teorías del crecimiento craneofacial: una revisión de literatura. UstaSalud. 2017;16:78-88. doi: https://doi.org/10.15332/us.v16i0.2022

17. Arias A, Espinal G, Ponce M, Posada A, Nava J, Salcedo B. Frecuencia de hábitos orales relacionados con la maloclusión en pacientes de 4 a 12 años: estudio comparativo entre San Luis de Potosí-México y Medellín-Colombia, 2016. Rev Nac Odontol. 2017;14(26). doi: https://doi. org/10.16925/od.v13i26.1814

18. Lima Illescas M, Rodríguez Soto A, García González B. Maloclusiones dentarias y su relación con los hábitos bucales lesivos. Rev Cuba Estomatol. 2019;56(2).

19. Díaz Méndez H, Ochoa Fernández B, Paz Quiñones L, Casanova Sales K, Coca García Y. Prevalencia de maloclusiones en niños de la escuela Carlos Cuquejo del municipio Puerto Padre, Las Tunas. Medisur. 2015;13(4).

20. Silva LCD, Vedovello SAS, Vedovello Filho M, Meneghin MC, Ambrosano Bovi GM, Degan W. Anxiety and oral habits as factors associated with malocclusion. Cranio. 2019:1-5. doi: https:// doi.org/10.1080/08869634.2019.1633492.

21. Sermet EÜ, Kocasarac HD, Elbay M, Kaya C, Baydemir C. Temporomandibular disorders and oral parafunction in children living with their parents and children living in institutional protective care: a comparative study. Int Dent J. 2016;67(1):20-8. doi: https://doi.org/10.1111/ idj. 12253

22. Węziak-Białowolska D, Białowolski P, McNeely E. The impact of workplace harassment and domestic violence on work outcomes in the developing world. World Dev [Internet]. 2020;126. doi: https://doi.org/10.1016/j.worlddev.2019.104732

23. Vishnoi P, Kambalyal P, Shyagali T, Bhayya D, Trivedi R, Jingar J. Age-wise and gender-wise prevalence of oral habits in 7-16-year-old school children of mewar ethnicity, india. Indian J Dent Sci. 2017;9(3):184-8. doi: https://doi.org/10.4103/IJDS.IJDS_33_17

24. Zakirulla M, Alshehri AD, Hudaybi AH, Fageeh SN, Alghothimi AA, Ali MG, et al. Oral habits: Prevalence and effects on occlusion among 7 to 13 years old school children in aseer, Saudi Arabia. Pesqui Bras Odontopediatria Clin Integr. 2020;20:1-9. doi: https://doi.org/10.1590/ pboci.2020.094 
12 Presencia de hábitos orales en pacientes con maloclusiones de 4 a 14 años, Cali, Colombia

25. Zapata Dávalos M, Lavado Torres A, Anchelia Ramírez S. Hábitos bucales y su relación con maloclusiones dentarias en niños de 6 a 12 años. Lima, Perú. KIRU. 2014;11(1):16-24.

26. Aloumi A, Alqahtani A, Darwish A. Oral parafunctional habits among preschool children in Riyadh, Saudi Arabia. Saudi J Oral Sci. 2018;5(1):22-7. doi: https://doi.org/10.4103/sjos. SJOralSci_46_17

27. S Dhull K, Verma T, Dutta B. Prevalence of Deleterious Oral Habits among 3- to 5-year-old Preschool Children in Bhubaneswar, Odisha, India. Int J Clin Pediatr Dent. 2018;11(3):210-3. doi: https://doi.org/10.5005/jp-journals-10005-1513. 